\title{
Evaluation of Emergency Health-Care Initiatives to Reduce Overcrowding in a Referral Medical Complex, Jeddah, Saudi Arabia
}

\author{
Khalid Alabbasi $^{\mathrm{a}} \quad$ Estie Kruger $^{\mathrm{b}}$ Marc Tennant ${ }^{\mathrm{b}}$ \\ aMinistry of Health, Jeddah, Saudi Arabia; 'bInternational Research Collaborative - Oral Health and Equity, \\ The University of Western Australia, Perth, WA, Australia
}

\section{Keywords}

Evaluations · Emergency health care · Overcrowding · Hospital patients · Saudi Arabia

\begin{abstract}
Purpose: Excessive delays and emergency department (ED) overcrowding have become an increasingly major problem for public health worldwide. This study was to assess the key strategies adopted by an ED, at a public hospital in Jeddah, to reduce delays and streamline patient flow. Materials and Methods: This study was a service evaluation for a Saudi patient population of all age-groups who attended the ED of a public hospital for the period between June 2016 and July 2019. The Saudi initiative to reduce the ED visits at the King Abdullah Medical Complex hospital has started on August 7, 2018. The initiative was to apply an urgency transfer policy which outlines the procedures to follow when patients arrive to the ED where they are reviewed based on the Canadian Triage and Acuity Scale (CTAS). Patients with less-urgent conditions (category 4 and 5) are referred to a primary healthcare practice (where a family medicine consultant is available). Patients with urgent conditions (category 1-3) are referred to a specialized health-care centre if the service is not currently provided. To test the effectiveness of ED initiative on reducing the overcrowd, data were categorized into before and after the initiative. The bivariate analysis $x^{2}$ tests and 2 sample $t$-tests were run to explore the relationship of gender and age with dependent variable emergency. Results: A
\end{abstract}

karger@karger.com www.karger.com/sjh

Karger!"
(C) 2021 The Author(s)

Published by S. Karger AG, Basel

This is an Open Access article licensed under the Creative Commons Attribution-NonCommercial-4.0 International License (CC BY-NC) (http://www.karger.com/Services/OpenAccessLicense), applicable to the online version of the article only. Usage and distribution for commercial purposes requires written permission. total of 233,998 patients were included in this study, $61.8 \%$ of them were males and the average age of ED patients were $35.5 \pm 18.6$ years. The majority of cases were those classified as "less urgent" (CTAS 4), which accounted for $65.4 \%$. Number of ED visits before and after the initiative was 67 and $33 \%$, respectively. ED waiting times after the initiative have statistically significantly decreased across all acuity levels compared to ED waiting times before the initiative. Conclusion and Implication: The findings suggest that the majority of patients arrive to the ED with less-urgent conditions and arrived by walking-in. The number of cases attending the ED significantly decreased following the introduction of the urgency transfer policy. Referral for less-urgent patients to primary healthcare centre may be an important front-end operational strategy to relieve congestion.

(c) 2021 The Author(s)

Published by S. Karger AG, Basel

\section{Introduction}

Emergency care is sought for acute medical conditions (i.e., heart attack or stroke) and serious injuries. However, excessive delays and emergency department (ED) overcrowding constitute the greatest threat to not only the quality of emergency care, but also has been associated with increased risk of errors, delayed time-critical care, increased morbidity, and excess deaths [1]. Overcrowding has become an increasingly major problem for public health worldwide. 
Fig. 1. Triage models used in the emergency department during the study period.

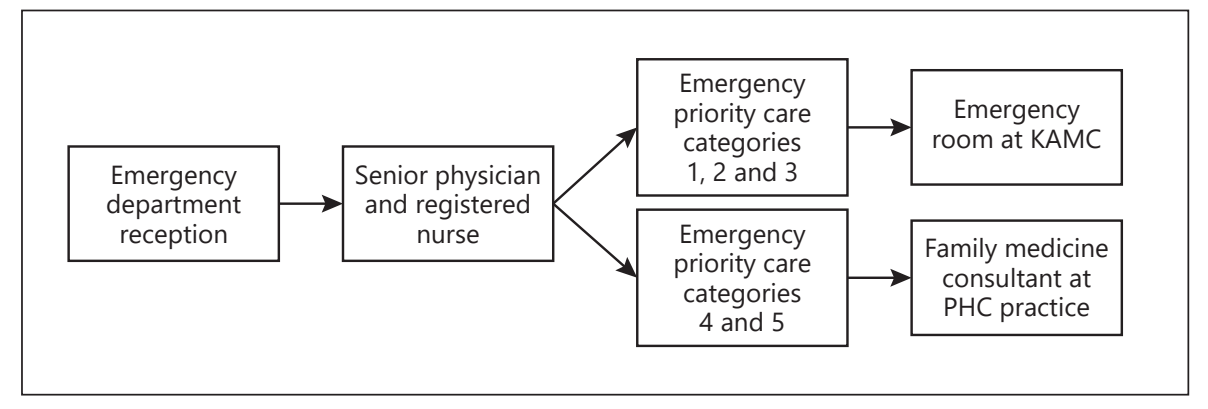

Table 1. Description of CTAS

\begin{tabular}{ll}
\hline Categories & Description \\
\hline Category 1 & $\begin{array}{l}\text { Immediately life-threatening patients - these patients should be seen by a treating doctor or nurse within } 2 \text { min of } \\
\text { arriving }\end{array}$ \\
\hline Category 2 & $\begin{array}{l}\text { Imminently life-threatening patients - these patients should be seen by a treating doctor or nurse within } 10 \text { min of } \\
\text { arriving }\end{array}$ \\
\hline Category 3 & $\begin{array}{l}\text { Potentially life-threatening patients - these patients should be seen by a treating doctor or nurse within } 30 \text { min of } \\
\text { arriving }\end{array}$ \\
\hline Category 4 & Potentially serious patients - these patients should be seen by a treating doctor or nurse within 60 min of arriving \\
\hline Category 5 & Less-urgent patients - these patients should be seen by a treating doctor or nurse within 120 min of arriving \\
\hline
\end{tabular}

CTAS, Canadian Triage and Acuity Scale.

Saudi EDs are experiencing an increasing demand for their services resulting in overcrowding and a high percentage of people wait well beyond the desirable period for receiving care. This might be due to a rising population or an increase in injury-related causes and chronic illnesses' complications. It has been reported that emergency services at the Saudi governmental hospitals are over-utilized for non-emergency cases [2]. In response to this issue, the Saudi government has implemented numerous schemes to address the complex causes of stress on EDs, and to meet the needs of modern health-care systems.

More recently, a nation-wide new model of care named "Urgent Care" was designed for patients who have an urgent need to see a clinician but whose illness or injury is not critical and does not require a visit to the ED and can be treated in a primary care setting. The "Urgent Care" model focuses mainly on streamlining the care pathway and making it more integrated and patient-centred which will improve patient experience outcomes (e.g., patients waiting times at ED), alleviate the burden on ED, and ensure financial sustainability (Table 2).

The model has been introduced by the King Abdullah Medical Complex (KAMC) in Jeddah through the physi- cian-led team triage services (Fig. 1), which requires that most patients presenting to Saudi EDs are reviewed based on the Canadian Triage and Acuity Scale (CTAS; Table 1) [3]. The patients are thereafter admitted, transferred to urgent care clinics or discharged from the department, in accordance with the level of urgency of the complaint. Patients with less-urgent conditions (category 4 and 5) are referred to a primary health-care practice - urgent care clinic (where a family medicine consultant is available). Patients with urgent conditions (category 1-3) are referred to a specialized health-care centre if the service is not currently provided. This patient throughput should allow for the efficient flow of patients in emergency room (ER), ensuring timely and appropriate levels of care.

The success of this change, based on reducing the number of people presenting to the EDs, addressing time delays, and improving the management of existing resources and patient flow, has not yet been assessed. Therefore, this study aimed to assess the transferred strategies adopted by an ED to reduce overcrowding and improve access to care, at a public hospital in Jeddah, Saudi Arabia. The study objectives were to ascertain whether the initiative of an urgency transfer policy makes a difference to 
Table 2. ED patient characteristics $(n=233,998)$

\begin{tabular}{|c|c|c|c|c|}
\hline \multirow[t]{2}{*}{ Variable } & \multirow[t]{2}{*}{ Total } & \multicolumn{2}{|l|}{ Arrivals } & \multirow[t]{2}{*}{$p$ value } \\
\hline & & before initiative & after initiative & \\
\hline Patients, $n(\%)$ & $233,998(100)$ & $156,859(67.0)$ & $77,139(33.0)$ & - \\
\hline Age, years, mean $\pm S D$ & $35.5 \pm 18.6$ & $35.2 \pm 18.4$ & $36.0 \pm 17.0$ & 0.001 \\
\hline \multicolumn{5}{|l|}{ Gender, $n(\%)$} \\
\hline Male & $144,585(61.8)$ & $97,331(41.6)$ & $47,286(20.2)$ & \multirow{2}{*}{0.001} \\
\hline Female & $89,413(38.2)$ & $59,577(25.5)$ & $29,859(12.8)$ & \\
\hline \multicolumn{5}{|c|}{ Type of emergency, $n(\%)$} \\
\hline CTAS 1 & $789(0.3)$ & $587(0.3)$ & $202(0.1)$ & \multirow{5}{*}{0.001} \\
\hline CTAS 2 & $3,559(1.5)$ & $2,462(1.1)$ & $1,137(0.5)$ & \\
\hline CTAS 3 & $54,360(23.2)$ & $35,981(15.4)$ & $18,379(7.9)$ & \\
\hline CTAS 4 & $152,166(65.0)$ & $98,089(41.9)$ & $54,077(23.1)$ & \\
\hline CTAS 5 & $23,084(9.9)$ & $19,740(8.4)$ & $3,344(1.4)$ & \\
\hline \multicolumn{5}{|c|}{ ED waiting time in minutes $($ mean $\pm S D)$} \\
\hline CTAS 1 & & $1.9 \pm 0.8$ & $1.8 \pm 0.65$ & 0.025 \\
\hline CTAS 2 & & $8.8 \pm 6.9$ & $6.5 \pm 1.9$ & 0.001 \\
\hline CTAS 3 & & $41.8 \pm 9.0$ & $22.9 \pm 4.9$ & 0.001 \\
\hline CTAS 4 & & $92.6 \pm 15.8$ & $46.5 \pm 9.4$ & 0.001 \\
\hline CTAS 5 & & $158.7 \pm 26.9$ & $92.6 \pm 15.8$ & 0.001 \\
\hline
\end{tabular}

CTAS, Canadian Triage and Acuity Scale; ED, emergency department.

the number of emergency visits at the selected hospital to reduce waiting times, and how, if at all, demographic characteristics are associated significantly with type of emergency.

\section{Materials and Methods}

\section{Study Area}

This study area was Jeddah city, which is the largest city in Makkah Province and Saudi Arabia's commercial capital, with a population of about 4 million people (as of 2017). The metropolitan area of the city is divided into 137 districts and has a population density of around 2,500 individuals per square kilometre [4].

This present study focussed on the KAMC in Jeddah. This complex is located in the north of Jeddah, covers an area of 266,000 square metres, and has a main 500-bed hospital with a planned total capacity of 1,100 beds.

\section{Study Design and Participants}

This study was a service review for a Saudi patient population of all age-groups who attended the ER of a public hospital for the period between June 2016 and July 2019. Patients' mode of attending the ER included walked in, transported by private care, or ambulance. The dataset was extracted from patients' monitoring forms in the hospital database (Excel). Data collected included age of the patient, gender, time of entering and leaving the service, and referral to an appropriate hospital. The Saudi initiative to reduce the ER visits at KAMC hospital has started on Aug 7, 2018. To test the effectiveness of ER initiative on reducing the overcrowd, data were categorized into before and after the initiative.

\section{Measures}

Emergency health-care initiatives to reduce overcrowding, which was the outcome of interest, in a referral medical complex was evaluated using the ED number of visits and ED waiting time.

\section{Data Analysis}

The statistical package for social sciences version 16 (SPSS Inc., Chicago, Il, USA) was used for data analysis. Data were screened for entry errors and outliers. Descriptive statistics was run to report the characteristics of the sample. The mean and standard deviation $(\mathrm{m} \pm \mathrm{SD})$ were reported for continuous variables and frequencies with percentages for categorical variables. The bivariate analysis $\chi^{2}$ tests and 2 sample $t$-tests were run to explore the relationship of gender and age with dependent variable (emergency types i.e., CTAS 1, CTAS 2, CTAS 3, CTAS 4, and CTAS 5). These latter were re-categorized to urgent (CTAS 1, CTAS 2, CTAS 3) and non-urgent (CTAS 4 and CTAS 5). The patients who died on arrival to ER were excluded from the analysis. A statistical significance was considered at $p<0.05$.

\section{Result}

The total number of patients who attended the ER at the KAMC between June 2016 and July 2019, was 239,790. Of these, 5,737 (2.4\%) had no data entered for the type of emergency and a further 55 patients were dead on arrival. A total of 233,998 patients were included in this study, with more males $(n=144,617 ; 61.8 \%)$ than females $(n=$ 89,$436 ; 38.2 \%$ ) (Table 2 ). The average age of ER patients 
Table 3. Factors associated with the type of ER urgency

\begin{tabular}{lrrr}
\hline Variable & Non-urgent & Urgent & $p$ value \\
\hline Age, years & $31.7 \pm 16$ & $45.9 \pm 21.0$ & $<0.0001$ \\
Gender, $n(\%)$ & & & \\
$\quad$ Male & $109,598(62.5)$ & $34,987(59.6)$ & $<0.0001$ \\
$\quad$ Female & $65,652(37.5)$ & $23,761(40.4)$ & \\
Arrivals, $n(\%)$ & & & \\
$\quad$ Before initiatives & $117,829(67.2)$ & $39,030(66.4)$ & $<0.0001$ \\
$\quad$ After initiatives & $57,421(32.8)$ & $19,718(33.6)$ & \\
\hline
\end{tabular}

ER, emergency room.

was $35.5( \pm 18.6)$ (normally distributed). The majority of cases were those classified as "less urgent" (CTAS 4), which accounted for $65.4 \%$. The commonality of other cases in order are $22.9 \%$ for "urgent" (CTAS 3), 9.8\% for "non-urgent" (CTAS 5), 1.4\% for "emergent" (CTAS 2), and $0.3 \%$ for "resuscitation" (CTAS 1). Number of ER visits before and after the initiative were 67 and 33\%, respectively. ED waiting times after the initiative have statistically significantly decreased across all acuity levels compared to ED waiting times before the initiative (6.5 vs. $8.8 \mathrm{~min}$ for CTAS 2 and 1.5 vs. $2.6 \mathrm{~h}$ for CATS 5, respectively).

\section{Bivariate Analysis Results of Factors Associated with Emergency Types}

Older patients were more likely to be classified as urgent cases than the younger counterparts and the differences was significant $(p<0.0001)$ (Table 3$)$. In addition, males were statistically significantly to report urgent arrivals (59.6\% males vs. $40 \%$ females).

\section{Transferred Cases}

Regardless of the level of urgency, the number of patients that arrived by walking-in can be seen to be far greater (95.5\%) than for other modes of arrival (Table 4). Between June 2016 and July 2019, a very small number of patients arrived by helicopter or police vehicle. The number transferred by ambulance was 10,238 patients, of which most was CTAS 3. CTAS 4 is the more common level of urgency for those who arrive by walk-in, police vehicle, and helicopter.

Transferred cases $(n=1,947)$ between June 2018 and July 2019 had statistical differences in relation to mean age, where older patients were more likely to be transferred due to non-urgent conditions than the younger ones $(14 \pm 15.7$ vs. $9.7 \pm 10.3, p=0.001)$ (Table 5). Females

Evaluation of Emergency Health-Care Initiative were more likely to be classified as urgent cases than males ( 47 vs. $41 \%$, respectively). However, no significant differences with respect to gender were observed $(p=$ $0.06)$.

\section{Discussion}

This study, conducted at the ED of the KAMC in Jeddah, gathered data on the presenting patients with respect to their age, gender, waiting time, mode of arrival, and referral details. To the best of our knowledge, this was the first small-scale study aimed to assess the transferred strategies adopted by an ED to reduce overcrowding and improve access to care, at a public hospital in Jeddah, Saudi Arabia. Our results reveal similarities to earlier studies showing that generally, overuse of ED services is high at the Ministry of Health hospitals in Jeddah. Both male gender and younger age are independent predictors for choosing ED, showing that urgency alone does not explain the difference in health-care services utilization (sometimes inappropriate). Similarly, the predominance of males and younger adult utilization of ED usually explained by the differences in health seeking behaviour, health knowledge sensitivity to symptoms, and willingness to report health problems. It also, might give an indicator of the community's make-up to accommodate specific health-care needs.

Our study findings are consistent with similar studies in Saudi Arabia where a large proportion (range 53$75.7 \%)$ of all ED visits for conditions that are less- or nonurgent (CTAS 4-5) [2,5]. Other studies outside Saudi Arabia (e.g., USA) have shown lesser percentages (rage between 30 and $50 \%$ ) of non-urgent conditions visiting the ED [6, 7]. Ideally, need should be the major determinant of health-care utilization; however, a non-urgent ED visit occurs when care is sought at an ED that could have been handled in a primary care setting. Studies have also shown a high percentage of medical service use (e.g., diagnostic and therapeutic interventions) when non-urgent cases being treated in the ED which will further strain the existing health-care system. Clearly, what drives the decision to seek ED care is that EDs are time convenient via "one-stop shops" that provide "total care" with relevant diagnostics, provided by a highly trained team in emergency medicine [8]. A study in 3 different public hospitals in Jeddah found a significant higher proportion of patients who were presented in the EDs with non-urgent conditions had not attempted to visit an outpatient clinic prior to visiting the $\operatorname{ED}(p=0.003)$ [2]. However, 
Table 4. Mode of arrival per type of emergency from June 2016 to July 2019 ( $n=230,983$ )

\begin{tabular}{lrrrrrr}
\hline Mode of arrival & CTAS 1 & CTAS 2 & CTAS 3 & CTAS 4 & CTAS 5 & Total* \\
\hline Ambulance & 449 & 1,525 & 6,012 & 2,109 & 143 & 10,238 \\
Helicopter & 1 & 1 & 1 & 2 & 0 & 5 \\
Police vehicle & 1 & 4 & 20 & 38 & 1 & 64 \\
Walk-in & 232 & 1,848 & 46,951 & 148,982 & 22,663 & 220,676 \\
Total & 683 & 3,378 & 52,984 & 151,131 & 22,807 & 230,983 \\
\hline
\end{tabular}

* Subjects with missing arrival data were not included.

Table 5. Transferred cases based on urgency from June 2018 to July $2019(N=1,947)$

\begin{tabular}{lclc}
\hline Variable & $\begin{array}{l}\text { Urgent } \\
(n=233)\end{array}$ & $\begin{array}{l}\text { Non-urgent } \\
(n=1,714)\end{array}$ & $p$ value \\
\hline $\begin{array}{l}\text { Age, mean } \pm \text { SD, years } \\
\begin{array}{l}\text { Gender, } n(\%) \\
\quad \text { Male }\end{array}\end{array}$ & $9.7 \pm 10.3$ & $14.9 \pm 15.7$ & $<0.0001$ \\
$\quad$ Female & $96(5)$ & $803(41)$ & 0.06 \\
\hline
\end{tabular}

the reasons behind the preference of ED over other option in our study are not known and further analysis is required.

Importantly, reductions are evident for the initiative, as all the number of cases after the initiative is lower than those before the initiative for all 5 types of emergencies, with CTAS 4 still being the dominant type of emergency. Children with less than critical conditions are more likely to be transferred to primary health-care services. This might suggest that triage ordering and transfers using strategies have shown to be effective particularly in dealing with non-urgent cases. The results show that a remarkable decrease in the number of ED visits after the implementation of the transferred strategies initiative, even when the number of transferred cases were nearly 2,000 cases. Certainly, this transferral strategies might at some extend increased the awareness level of the community regarding other option (such as primary care centres), namely, when the majority of the cases arrived at ED by walking-in.

The current study reported a relatively higher average waiting time before the initiative ( $8.8 \mathrm{~min}$ for CTAS 2 and $2.6 \mathrm{~h}$ for CATS 5). This waiting time is comparable with other data from Saudi Arabia (Makkah), for example, where the average waiting time in ED was $3 \mathrm{~h}$ [9]. However, diverting non-urgent patients to primary health- care centres has significantly decrease the ED waiting time, since many experience extended periods in ED waiting rooms. The difference between the average ED waiting time before and after the initiative is nearly half across most of the urgency categories with CATS 1 patients had the lowest waiting time, while CTAS 5 patients had the longest waiting time. Despite this improvement, the initiative should therefore be consolidated at the institution investigated with a combined commitment to improving community awareness and enhancing the primary care services to ensure sustainability.

The emergency care pathway is multifaceted, with patients presenting to an ED through emergency ambulances, walk-in, or referral by a primary health-care provider. This study indicated that the vast majority of patients arrived at the ED by walking-in. The literature reports an enormous variability on the percentage of walk-ins to EDs (ranging from 6 to 80\%) who could have been seen by a general practitioner [10-13]. Although patients with urgent need (CTAS 1-2) were more likely to arrive by ambulance. Unfortunately, a prominent proportion of the patients presented to ED with critical conditions arrived by walking-in. Similar finding was reported elsewhere [14]. The efficacy of urgent conditions management is very time dependant, and it seems that a significant proportion of the population is not aware of the critical conditions. Therefore, extensive public education regarding early recognition of critical and non-critical cases and to call 997 to receive ambulance transport is vital.

\section{Strength and Limitation}

This study was conducted over a 3 -year period and the number of cases was large, including over 23,000 patients. However, it was limited to a single hospital. The study would have to be broadened to include more institutions so that generalizations can be made for Saudi hospitals overall, and by including more data targeted at improving emergency health-care. 


\section{Implications and Recommendations}

Most of the crowding in an ED may involve low-acuity patients like those with minor injuries or minor illnesses. Therefore, referral for such patients to primary healthcare centre may be an important front-end operational strategy to relieve congestion. More attention should be given to modifiable features of primary health care that have the potential to help reduce ED use. Additionally, as a long-term strategy, expanding the family physician program, establishing integrated health records, and improving referral system across the country should be considered as a national priority. Finally, clear national urgent care guidelines are vital to enable effective triage of cases.

\section{Conclusion}

The findings suggest that the majority of patients arrive to the ER with less-urgent conditions and arrived by walking-in. The number of cases attending the ER significantly decreased following the introduction of the urgency transfer policy. Children with less-critical conditions are more likely to be transferred to primary healthcare services. The present study has potential significance because it will allow for an in-depth case study-based investigation of health-care initiatives at other emergency health-care institutions throughout Saudi Arabia. It could also be an indicative as to how well Saudi Arabia is progressing in terms of the provision of health-care services.

\section{Acknowledgements}

The authors would like to thank Professor John McGeachie and Dr. Saba Kassim for their continuous ongoing support of the whole team.

\section{Statement of Ethics}

This study protocol was submitted to the Human Research Ethics Committee at the University of Western Australia (RA/4/20/6317) and Ministry of Health in Saudi Arabia (H02-J-002) for review and approval. The Ethics Committee waived the need for informed patient consent as this study involved anonymous secondary data extracted from the hospital medical records. The research was conducted ethically in accordance with the World Medical Association Declaration of Helsinki.

\section{Conflict of Interest Statement}

The author declared no potential conflicts of interest.

\section{Funding Sources}

This research received no specific grant from any funding agency in the public, commercial, or not-for-profit sectors.

\section{Author Contributions}

Conceptualization: K.A., M.T. and E.K.; methodology: K.A. and E.K.; software: K.A. and M.T.; formal analysis: K.A. and E.K.; data collection,:K.A.; writing-original draft preparation: all authors; writing-review and editing: K.A., E.K., and M.T.; and all authors have approved the final manuscript.

\section{References}

1 Di Somma S, Paladino L, Vaughan L, Lalle I, Magrini L, Magnanti M. Overcrowding in emergency department: an international issue. Intern Emerg Med. 2015;10(2):171-5.

2 Dawoud SO, Ahmad AM, Alsharqi OZ, AlRaddadi RM. Utilization of the Emergency department and predicting factors associated with its use at the Saudi Ministry of Health General Hospitals. Glob J Health Sci. 2015; 8(1):90-106.

3 Triage Training Resources. The Canadian triage and acuity scale: Combined adult/paediatric educational program. Canadian Association of Emergency Physicians. Available from: http://ctasphctas.ca/wpcontent/uploads/2018/05/participant_manual_v2.5b_ november_2013_0.pdf. Accessed 2019 Nov 15.

4 World Population Review. Jeddah population 2019. World Population Review. Available from: http://worldpopulationreview.com/ world-cities/jeddah-population/. Accessed 2019 Nov 15.
5 Al-Otmy SS, Abduljabbar AZ, Al-Raddadi RM, Farahat F. Factors associated with non-urgent visits to the emergency department in a tertiary care centre, western Saudi Arabia: cross-sectional study. BMJ Open. 2020;10:e035951.

6 McCormack LA, Jones SG, Coulter SL. Demographic factors influencing nonurgent emergency department utilization among a Medicaid population. Health Care Manag Sci. 2017;20:395-402.

7 Uscher-Pines L, Pines J, Kellermann A, Gillen E, Mehrotra A. Emergency department visits for nonurgent conditions: systematic literature review. Am J Manag Care. 2013;19(1): 47-59.

8 Lowthian JA, Curtis AJ, Cameron PA, Stoelwinder JU, Cooke MW, McNeil JJ. Systematic review of trends in emergency department attendances: an Australian perspective. Emerg Med J. 2011;28(5):373-7.

9 Bukhari H, Albazli K, Almaslmani S, Attiah A, Bukhary E, Najjar F, et al. Analysis of waiting time in emergency department of Al-
Noor specialist Hospital, Makkah, Saudi Arabia. Open J Emerg Med. 2014;2(4):67-73.

10 Lee A, Lau FL, Hazlett CB, Kam CW, Wong P, Wong TW, et al. Factors associated with non-urgent utilization of Accident and Emergency services: a case-control study in Hong Kong. Soc Sci Med. 2000;51(7):1075-85.

11 Coleman P, Irons R, Nicholl J. Will alternative immediate care services reduce demands for non-urgent treatment at accident and emergency? Emerg Med J. 2001;18(6):482-7.

12 Snooks H, Williams S, Crouch R, Foster T, Hartley-Sharpe C, Dale J. NHS emergency response to 999 calls: alternatives for cases that are neither life threatening nor serious. BMJ. 2002;325(7359):330-3.

13 Van Uden CJ, Crebolder HF. Does setting up out of hours primary care cooperatives outside a hospital reduce demand for emergency care? Emerg Med J. 2004;21(6):722-3.

14 Mohammad YM. Mode of arrival to the emergency department of stroke patients in the United States. J Vasc Interv Neurol. 2008;1(3):83-6.
Evaluation of Emergency Health-Care Initiative
Saudi J Health Syst Res 2021;1:134-139 DOI: 10.1159/000517487 